\title{
Review of: "Leveraging long short-term memory (LSTM)-based neural networks for modeling structure-property relationships of metamaterials from electromagnetic responses"
}

\section{Xuhui Bu}

Potential competing interests: The author(s) declared that no potential competing interests exist.

In this report, a neural network modeling method was proposed to predict the response of MMs and addressed the design parameters for a desired spectral behavior. The problem is clearly stated, and the results verify the effectiveness of the method, but the experimental results only give some textual descriptions, lacking mathematical descriptions of the specific structure of the proposed network prediction model and neural network model, and seem to lack persuasiveness. It is recommended to add it. On the whole, the content of this report is correct, and it is recommended to be accept after minor revised. 